\title{
A multifunctional $\beta$-cyclodextrin-conjugate photodelivering nitric oxide with fluorescence reporting
}

\author{
Gábor Benkovics ${ }^{\mathrm{b}, \mathrm{c}, 1}$, Marta Perez-Lloret ${ }^{\mathrm{a}, 1}$, Damien Afonso ${ }^{\mathrm{a}}$, András Darcsi ${ }^{\mathrm{d}}$, \\ Szabolcs Béni ${ }^{\mathrm{d}}$, Éva Fenyvesi ${ }^{\mathrm{b}}$, Milo Malanga ${ }^{\mathrm{b}, *}$, Salvatore Sortino ${ }^{\mathrm{a}, *}$ \\ a Laboratory of Photochemistry, Department of Drug Sciences, University of Catania, I-95125, Italy \\ ${ }^{\mathrm{b}}$ CycloLab, Cyclodextrin RE'D Ltd, Illatos út 7, H-1097 Budapest, Hungary \\ ${ }^{\mathrm{c}}$ Department of Organic Chemistry, Faculty of Science, Charles University in Prague, Hlavová 8, 12843 Prague 2, Czech Republic \\ d Department of Pharmacognosy, Semmelweis University, Üllői út 26, H-1085, Hungary
}

A R T I C L E I N F O

\section{Article history:}

Received 25 March 2017

Received in revised form 9 May 2017

Accepted 10 May 2017

Available online 11 May 2017

\section{Keywords:}

Cyclodextrin

Nitric oxide

Singlet oxygen

Light

Fluorescence

\begin{abstract}
A B S T R A C T
This contribution reports the design, synthesis and photochemical properties of a novel cationic, water soluble, $\beta$-cyclodextrin $(\beta C D)$ conjugate integrating an anthracene moiety and a nitroaniline derivative within the primary side of the $\beta C D$ scaffold. Photoinduced energy transfer between the anthracene and the nitroaniline chromophores effectively suppresses the fluorescence of the anthracene unit. Excitation with visible light triggers the release of nitric oxide (NO) from the nitroaniline chromophore, accompanied to the concomitant revival of the anthracene fluorescence, which acts as an optical reporter for detecting the amount of the NO released. Furthermore, the anthracene moiety photogenerates singlet oxygen $\left({ }^{1} \mathrm{O}_{2}\right)$ sequentially to NO release. The conjugate is also able to accommodate hydrophobic guests within the $\beta C D$ cavity, as proven by using naphthalene as a model compound. In view of the key role NO and ${ }^{1} \mathrm{O}_{2}$ play as anticancer and antibacterial species, the present $\beta C D$ derivative represents an intriguing candidate for further studies in biopharmaceutical research addressed to multimodal therapeutic applications.
\end{abstract}

(C) 2017 Elsevier B.V. All rights reserved.

\section{Introduction}

Cyclodextrins (CDs) have been greatly developed during the last thirty years as carriers of "conventional" drugs (Davis and Brewster, 2004; Loftsson and Douchene, 2007). However, the use of $C D$ s as suitable vehicles for photoactivable therapeutic compounds has been only recently object of attention (Mazzaglia et al., 2012). Due to their hydrophobic cavity, natural CDs can host a variety of photosensitive agents by supramolecular interactions (Bortolus and Monti, 1996; Monti and Sortino, 2002). Nevertheless, in most cases, the low binding constants between unmodified CDs and guest molecules represent a major drawback of these systems as bio-carriers, making the modification of the CD structure strictly necessary in view of actual applications (Mazzaglia et al., 2006). Modification of the CDs molecular scaffold through functionalization of the primary and/or secondary hydroxyl groups with

\footnotetext{
* Corresponding authors.

E-mail addresses: malanga@cyclolab.hu (M. Malanga), ssortino@unict.it (S. Sortino).

1 These authors contributed equally to this work.
}

suitable photoresponsive units allows to obtain multifunctional nanocarriers with intriguing properties whilst, at the same time, maintaining the macrocycle's capacity for guests encapsulation (Mourtzis et al., 2007, 2008; Yannakopoulou et al., 2011).

Nitric oxide (NO) is a pleiotropic bioregulator of important physiological and pathophysiological processes encompassing neurotransmission, vasodilatation and hormone secretion in living bodies (Ignarro, 2010). Besides, NO is an excellent sacrificial antioxidant, anticancer and antibacterial agent (Ignarro, 2009). This scenario has opened a fervent research activity devoted to developing compounds able to release NO under physiological conditions as potential pharmaceuticals to fight a variety of diseases (Wang et al., 2002, 2005; Riccio and Schoenfisch, 2012; Seabra and Durán, 2010). However, the biological effects of NO are strictly depending on its concentration, location and dose (Jia et al., 2002). This has made the light-activated NO donors very appealing due to the superb spatiotemporal accuracy the light triggering offers (Ford, 2008; Sortino, 2010; Fry and Mascharak, 2011; Ford, 2013). Several NO photodonors (NOPD) have been supramolecularly combined with CDs derivatives (Fraix et al., 2016). In contrast, 
only limited examples of NO photodonor covalent conjugates with CDs are reported to date (Piras et al., 2013).

Quantification of the NO delivery in real time is another important issue to be faced, especially when the main interest is to reach a critical molecule concentration to induce a specific effect. A suitable way to address this quantification task is based on the use of a fluorescent reporter. This elegant strategy relies on the simultaneous photorelease of the desired bioactive species, i.e. NO, and a fluorescent component (the reporter) from the same nonfluorescent precursor (Veldhuyzen et al., 2003; Pellois et al., 2004; Weinstain et al., 2010). The release process can be thus easily quantified by monitoring the fluorescence emission of the reporter, which acts exactly as an optical counter of the bioactive species. Recently, we have reported interesting properties of an ad-hoc designed molecular conjugate integrating two chromogenic centers within the same covalent skeleton (Vittorino et al., 2011; Kirejev et al., 2014), an anthracene moiety and a nitroaniline derivative, this latter as a suitable NOPD (Caruso et al., 2007) In this compound, the typical emission of the anthracene fluorophore is completely suppressed by a photoinduced energy transfer to the nitroaniline moiety. We demonstrated that the photorelease of NO leads to the revival of the fluorescence of anthracene fluorophore which acts as a fluorescent reporter for the NO delivery in living cells (Vittorino et al., 2011; Kirejev et al., 2014).

Based on the above scenario we considered it of value to translate this NO photorelease with fluorescent reporting concept in a novel conjugate, which covalently integrates the NO photoreleaser and the anthracene moiety within the same positively charged $\beta C D$ scaffold. This contribution reports the synthesis, characterization and photochemical properties of the cationic $\beta C D$ conjugate AN- $\mathbf{B C D}$-NOPD and the suitable model compounds AN$\boldsymbol{\beta C D}$ and $\boldsymbol{\beta C D}-\mathrm{NOPD}$ (Fig. 1).

\section{Experimental}

\subsection{Chemicals}

6-Monodeoxy-6-monoazido- $\beta C D$ and 6-monotosyl- $\beta C D$ are fine chemical products of CycloLab, 1,3-diaminopropanol ( $\geq 99 \%$ ), 4-nitro-3-(trifluoromethyl)aniline (98\%), glycidyltrimethylammonium chloride (technical grade, $\geq 90 \%$ ), sodium azide (ReagentPlus ${ }^{\circledR}, \geq 99.5 \%$ ), hydrazine monohydrate (reagent grade, $98 \%)$ sodium borohydride ( $\geq 98.0 \%)$, anthracene-9- carboxaldehyde $\quad(\geq 97.0 \%), \quad$ p-toluenesulfonyl chloride (ReagentPlus ${ }^{\circledR}, \geq 99 \%$ ), trimethylamine ( $\geq 99 \%$ ), 1,3-propanedithiol ( $\geq 99 \%$ ), palladium on carbon (5\%), were sourced from SigmaAldrich.

Dimethyl sulfoxide, formic acid, methanol, pyridine were obtained from Molar Chemicals.

\subsection{Instrumentation}

${ }^{1} \mathrm{H},{ }^{13} \mathrm{C}$ NMR spectra and DEPT-ed-HSQC spectra were recorded in DMSO- $d_{6}$ or $\mathrm{D}_{2} \mathrm{O}(10 \mathrm{mg}$ dissolved in $0.8 \mathrm{~mL}$ of deuterated solvent) on a Varian DDR-600 spectrometer at $600 \mathrm{MHz}$ at $298 \mathrm{~K}$.

Mass spectra were obtained on Bruker ESQUIRE 3000 ES-ion trap instrument with electrospray ionization (ESI) in negative mode. All samples were dissolved in water.

Preparative chromatographic separations were performed on a Büchi preparative chromatography system using SiliCycle Silica Cartridge $(4 \mathrm{~cm} \times 15 \mathrm{~cm})$ packed with Lichroprep $(120 \mathrm{~g})$ RP-18 Phase $(40-63 \mu \mathrm{m})$ as a stationary phase and water-methanol gradient elution. The chromatographic station was equipped with Büchi UV Photometer C-635 as a detector (detection wavelength set at $390 \mathrm{~nm}$ for NOPD appended derivatives and at $245 \mathrm{~nm}$ for the tosylated intermediate).

UV-vis absorption and fluorescence spectra were recorded with a Jasco V 650 spectrophotometer and a Fluorolog-2 (Model, F111) spectrofluorimeter, respectively. Fluorescence lifetimes were recorded with the same spectrofluorimeter equipped with a TCSPC Triple Illuminator. The samples were irradiated by a pulsed diode excitation source Nanoled at $370 \mathrm{~nm}$. The kinetic was monitored at $420 \mathrm{~nm}$ and each solution itself was used to register the prompt at $370 \mathrm{~nm}$. The system allowed measurement of fluorescence lifetimes from 200 ps. The multiexponential fit of the fluorescence decay was obtained using the following equations: $I$ $(t)=A_{1} \exp \left(^{-t / \tau 1}\right)+\mathrm{A}_{2} \exp \left(^{-t / \tau 2}\right)$ (for the biexponential fit) and $I$ $(t)=A_{1} \exp \left({ }^{-t / \tau 1}\right)+A_{2} \exp \left({ }^{-t / \tau 2}\right)+A_{3} \exp \left(^{-t / \tau 3}\right)$ (for the triexponential fit). Multiexponential fitting were used because they gave much better chi-square values than monoexponential fitting.

${ }^{1} \mathrm{O}_{2}$ emission was registered with the same spectrofluorimeter as above equipped with a NIR-sensitive liquid nitrogen cooled photomultiplier, exciting the air-equilibrated samples in $\mathrm{D}_{2} \mathrm{O}$ solution at $380 \mathrm{~nm}$ with the fluorimeter lamp.

Photolysis of the samples in solution was performed in a thermostated quartz cell ( $1 \mathrm{~cm}$ pathlength, $3 \mathrm{~mL}$ capacity) under

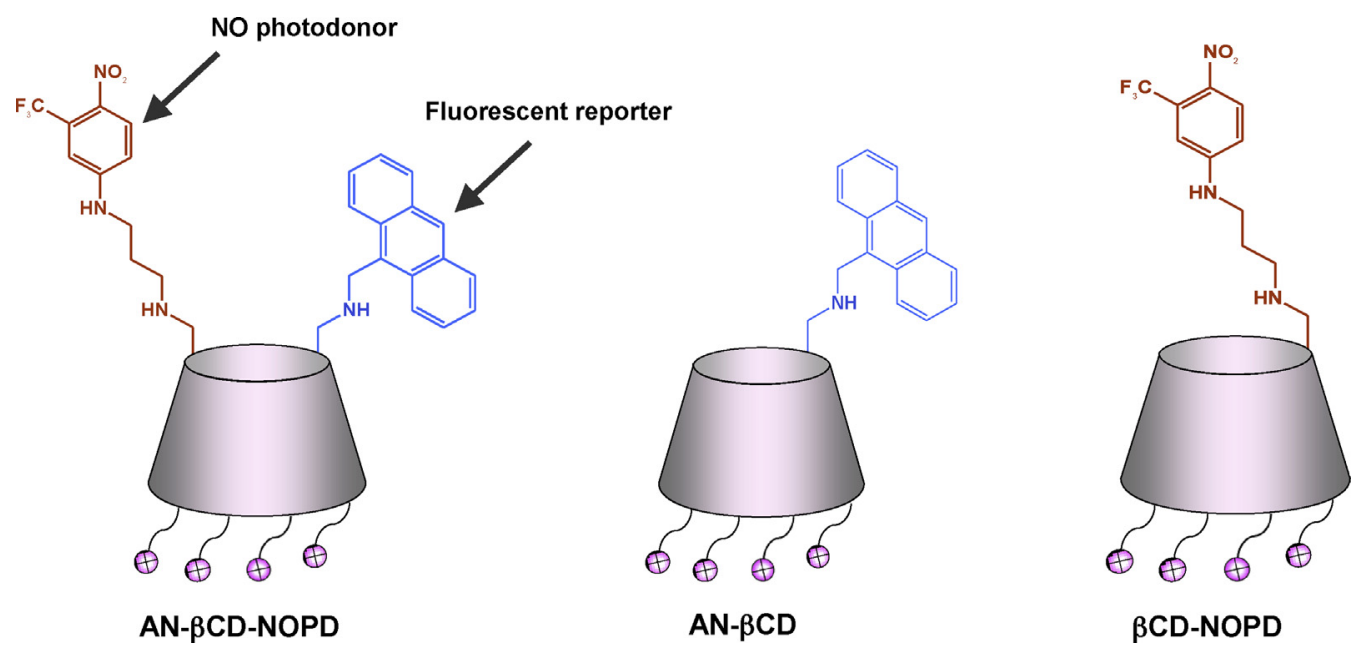

Fig. 1. Schematic of the multifunctional $\beta$ CD conjugate AN- $\boldsymbol{\beta C D}-\mathbf{N O P D}$ and the model compounds AN- $\boldsymbol{\beta C D}$ and $\boldsymbol{\beta C D}-\mathbf{N O P D}$. 

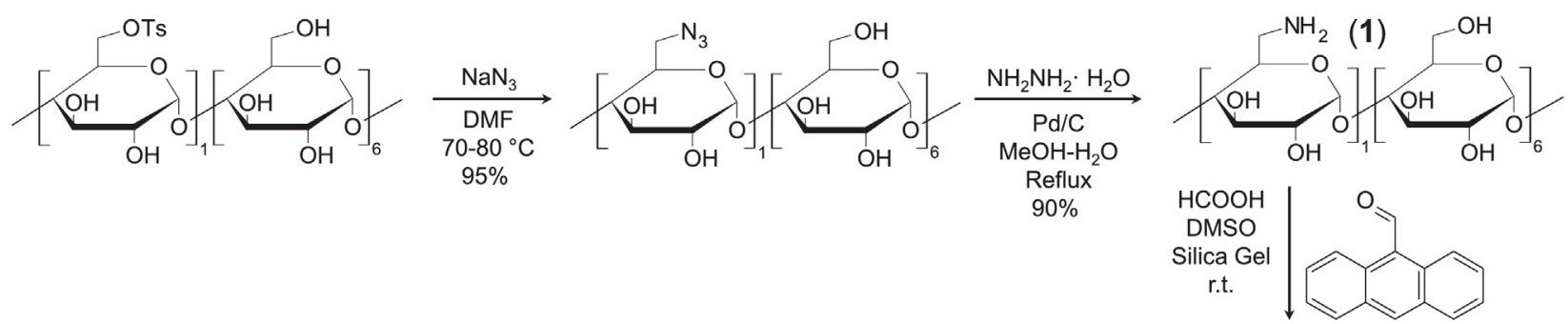

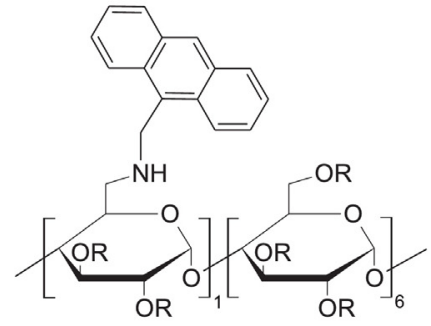

$\mathrm{R}=-\mathrm{H}$ or $-\mathrm{CH}_{2} \mathrm{CHOHCH}_{2}-\mathrm{N}\left(\mathrm{CH}_{3}\right)_{3} \mathrm{Cl}$

AN- $\beta$ CD

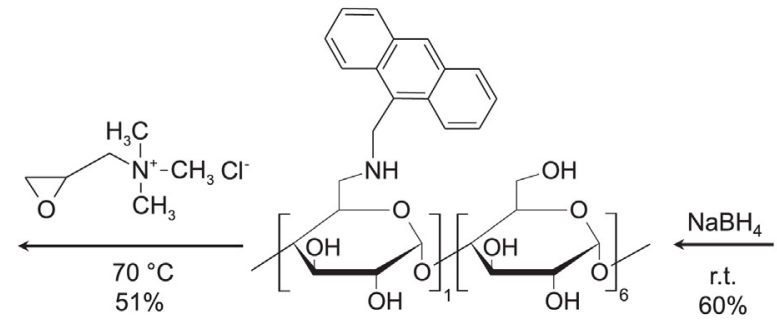

(2)

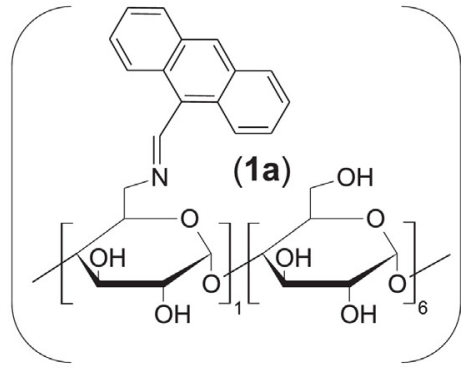

Scheme 1. Reaction scheme for the synthesis of AN- $\beta \mathbf{C D}$.

gentle stirring, by using a continuum laser with $\lambda_{\text {exc }}=405 \mathrm{~nm}$ (ca. $100 \mathrm{~mW}$ ) having a beam diameter of ca. $1.5 \mathrm{~mm}$.

NO release was measured with a World Precision Instrument, ISO-NO meter, equipped with a data acquisition system, and based on direct amperometric detection of NO with short response time ( $<5 \mathrm{~s}$ ) and sensitivity range $1 \mathrm{nM}-20 \mu \mathrm{M}$. The analog signal was digitalized with a four-channel recording system and transferred to a PC. The sensor was accurately calibrated by mixing standard solutions of $\mathrm{NaNO}_{2}$ with $0.1 \mathrm{M} \mathrm{H}_{2} \mathrm{SO}_{4}$ and $0.1 \mathrm{M} \mathrm{KI}$ according to the reaction:

$4 \mathrm{H}^{+}+2 \mathrm{I}^{-}+2 \mathrm{NO}_{2}^{-} \rightarrow 2 \mathrm{H}_{2} \mathrm{O}+2 \mathrm{NO}+\mathrm{I}_{2}$

Irradiation was performed in a thermostated quartz cell $(1 \mathrm{~cm}$ path length, $3 \mathrm{~mL}$ capacity) under gentle stirring by using the same $405 \mathrm{~nm}$ laser sources described above. NO measurements were carried out with the electrode positioned outside the light path in

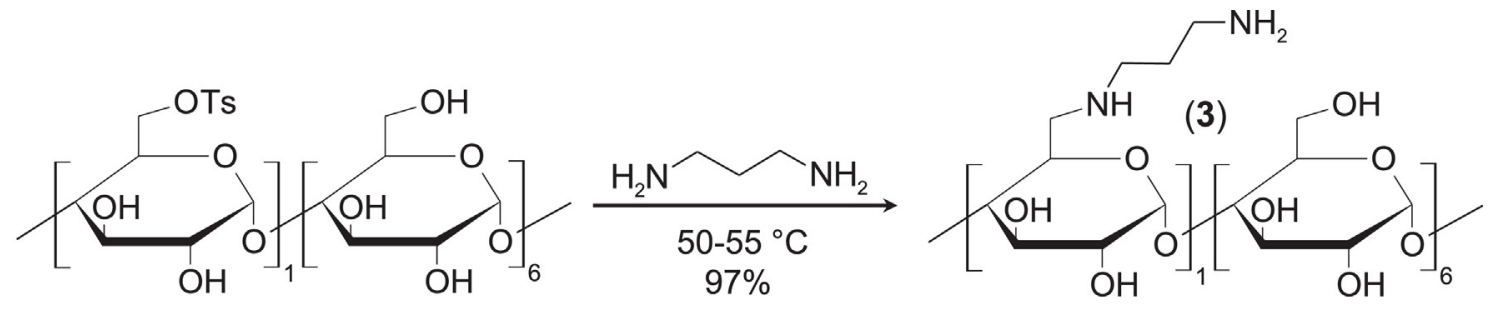

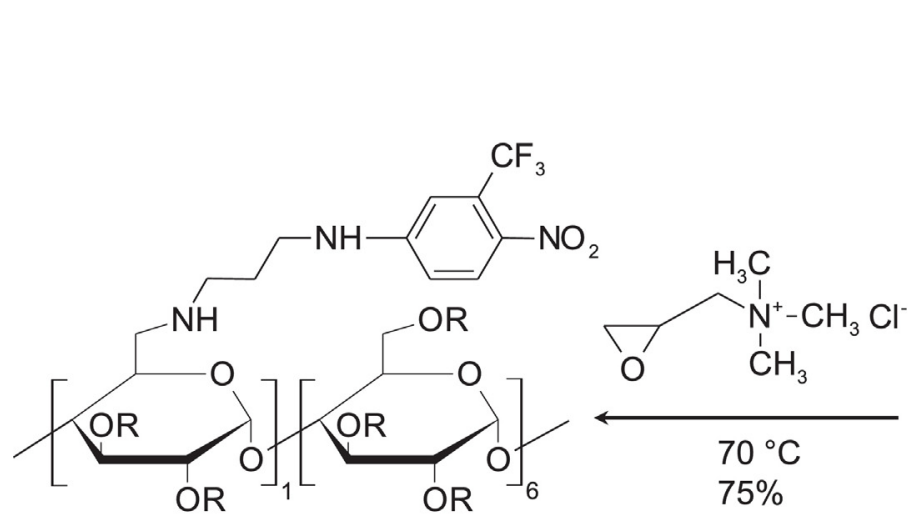<smiles>O=[N+]([O-])c1ccc(Cl)cc1C(F)(F)F</smiles>

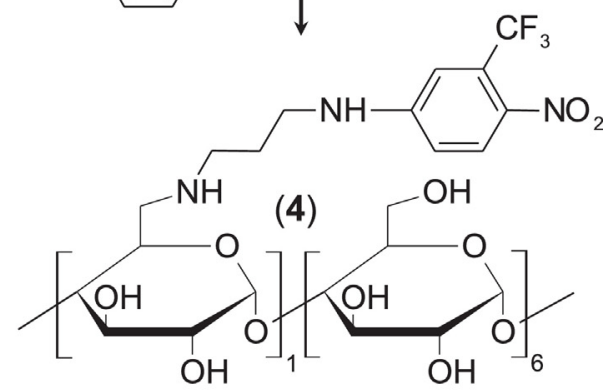

$\mathrm{R}=-\mathrm{H}$ or $-\mathrm{CH}_{2} \mathrm{CHOHCH}_{2}-\mathrm{N}\left(\mathrm{CH}_{3}\right)_{3} \mathrm{Cl}$

BCD-NOPD

Scheme 2. Reaction scheme for the synthesis of $\boldsymbol{\beta C D}$-NOPD. 

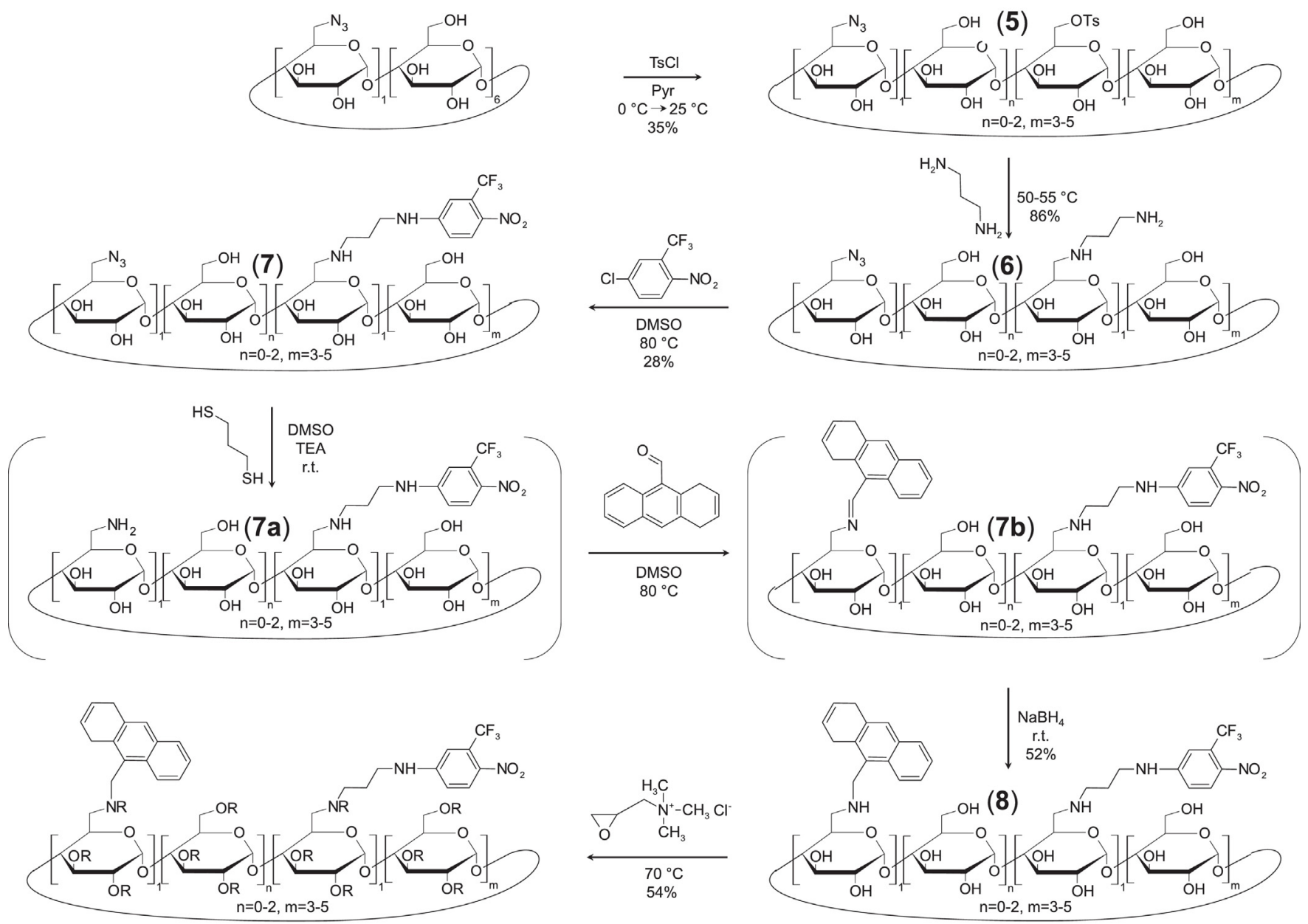

$\mathrm{R}=-\mathrm{H}$ or $-\mathrm{CH}_{2} \mathrm{CHOHCH}_{2}-\mathrm{N}\left(\mathrm{CH}_{3}\right)_{3} \mathrm{Cl}$

AN-BCD-NOPD

Scheme 3. Reaction scheme for the synthesis of AN-BCD-NOPD.

order to avoid false NO signal due to photoelectric interference on the ISO-NO electrode.

\section{Results and discussion}

\subsection{Syntheses}

The model compound AN- $\boldsymbol{B C D}$ was synthesized according to the procedure illustrated in Scheme 1 and briefly described in the following (the full details are reported in the Supporting Information).

6-Monotosyl- $\beta C D$ was promptly converted to the aminoanalog 1 by exhaustive reduction of the intermediate 6-monodeoxy-6-monoazido $\beta C D$, according to literature (Jicsinszky and Iványi, 2001). The amino group, located on the primary rim of CD scaffold, is a suitable anchoring point for the introduction of the anthracene chromophore. As compound $\mathbf{1}$ is obtained as free base, the coupling could be achieved by a nucleophilic substitution in DMF with the commercially available 9-(chloromethyl)anthracene. This reaction setting was explored, but the conversion rate was not satisfactory ( $\sim 30 \%$ formation of compound 2 ) and $\mathrm{N}$ dialkylated product ( $\mathrm{CD}$ scaffold containing two anthracene moieties) was considerably formed under the tested experimental conditions ( $\sim 10 \%$ ). Although chromatographic purification of the crude can be attempted, the procedure is time-consuming, not effective and resulted in low-yield. As efficient alternative for the preparation of compound (2) a two-steps one-pot reaction based on imine-reduction, allowing good conversion rate and avoiding chromatographic purification, was applied. The addition of silica gel to the reaction mixture during the first step was a fundamental requirement for achieving quantitative conversion to the imine intermediate 1a. The nano-porous material was effective in removing the water accumulated during the condensation process thus making convenient the coupling reaction. The second step based on the mild reducing agent sodium borohydride proceeded quantitatively within expectations. The introduction of the permanent charges on the $\mathrm{CD}$ scaffolds was achieved by using the alkylating agent, glycidyltrimethylammonium chloride, as reaction solvent. This approach has the advantages of minimizing the by-products formation and reducing the work-up to simple fractional crystallization cycles.

The model compound $\boldsymbol{\beta C D}$-NOPD was synthesized according to the procedure illustrated in Scheme 2 and briefly described in the following (the full details are reported in the Supporting Information).

The flexible linker, 1,3-diaminopropane, was effectively introduced on the $C D$ scaffold by nucleophilic substitution on the 6monotosyl- $\beta C D$. In order to reduce the formation of by-products such as $C D$ dimers (two or more $C D$ units connected through the linker) and 3,6-anhydro derivatives, 1,3-diaminopropane was used 


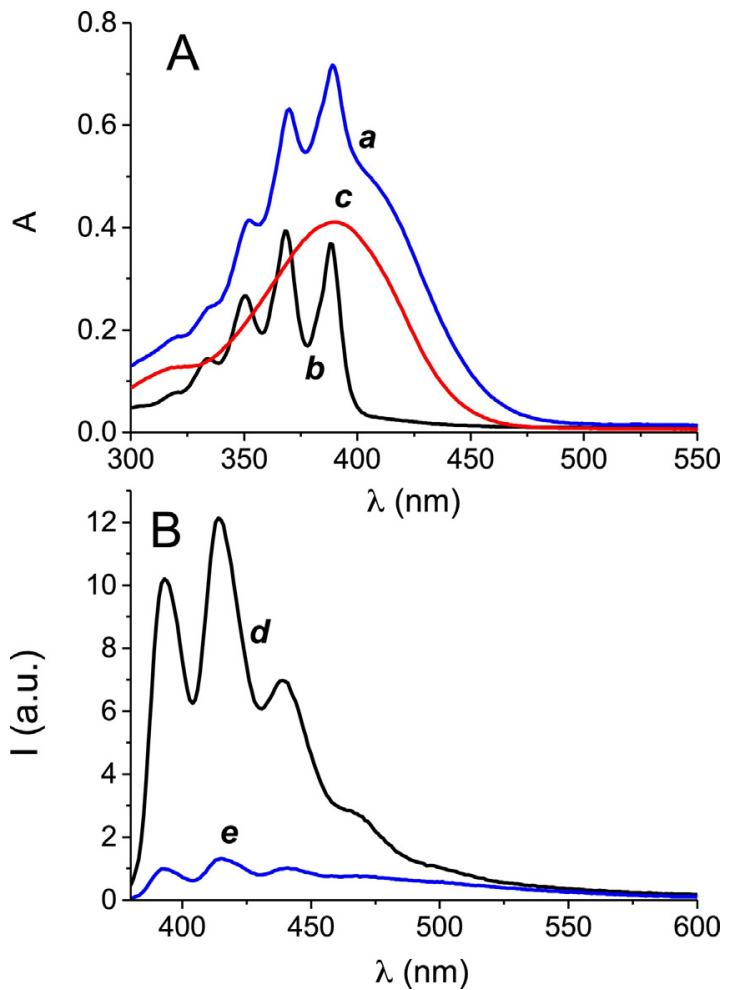

Fig. 2. (A) Absorption spectra of $40 \mu \mathrm{M}$ aqueous solution AN-BCD-NOPD (a) and the model compounds AN- $\boldsymbol{\beta C D}(\boldsymbol{b})$ and $\boldsymbol{\beta C D}-\mathbf{N O P D}(\boldsymbol{c})$. (B) Fluorescence emission

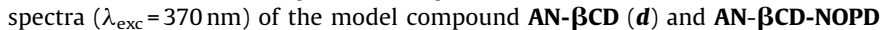
(e).

in large excess (reaction solvent) and 6-monotosyl- $\beta C D$ was added portion-wise to the warm solvent solution. These precautions ensured that 6 -monotosyl- $\beta C D$ was always acting as limiting reagent and promptly converted to 6-monodeoxy-6-mono-(3aminopropylamino) $\beta C D$. The large excess of 1,3-diaminopropane in combination with an adequate work-up (evaporation under reduced pressure and precipitation with methanol-diisopropyl ether mixture) also maintained compound $\mathbf{3}$ in free base form. This is a strict requirement to successfully accomplish the following reaction step as no extra base can be added to the reaction mixture due to the high reactivity of 4-chloro-1-nitro-2-(trifluoromethyl) benzene toward these species. It is worth to mention that the decision of attaching the NOPD moiety to the CD scaffold through a

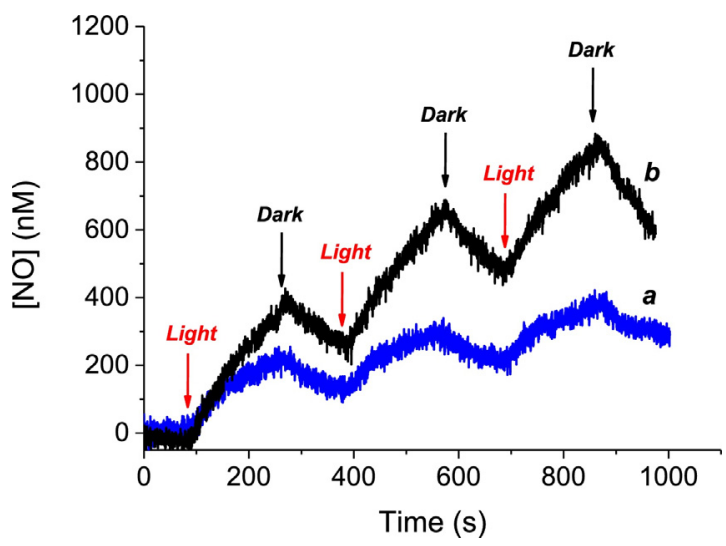

Fig. 3. NO release profile observed for optically matched aqueous solutions of ANBCD-NOPD (a) and $\boldsymbol{\beta C D}$-NOPD $(\boldsymbol{b})$ upon irradiation with $\lambda_{\text {exc }}=405 \mathrm{~nm}$ at $25^{\circ} \mathrm{C}$. linker, was the result of several unsuccessful attempts to directly introduce the chromophore on the CD rim. No adequate reaction conditions were found to react the reagent 4-chloro-1-nitro-2(trifluoromethyl)benzene with the free base of 6-monoamino-6monodeoxy- $\beta C D$. The most probable reason is that the nitrobenzene derivative formed a strong inclusion complex with the $\beta C D$ cavity locating the chlorine atom on the secondary side (Ueno and Breslow, 1982). The establishment of this supramolecular interaction precludes primary-side substitution.

Compound 4 was obtained in moderate yield by reacting the free base of compound $\mathbf{3}$ with 4-chloro-1-nitro-2-(trifluoromethyl) benzene. As no extra base could be added to the reaction mixture without consuming the chromophore, the product was formed only at around $40 \%$ conversion rate. Reversed-phase chromatographic purification was needed to separate the unreacted compound $\mathbf{3}$ from the target product. The solubility profile of compound $\mathbf{4}$ was improved by modifying some of the hydroxyl groups with permanently charged side-chains.

The multifunctional conjugate AN-BCD-NOPD was synthesized according to the procedure illustrated in Scheme 3 and briefly described in the following (the full details are reported in the Supporting Information). In order to introduce both the chromophores on the primary side of the CD scaffold, tosylation of 6monoazido-6-monodeoxy- $\beta C D$ was performed in pyridine. This approach is selective for the modification of the primary rim of the macrocycle (Matsui et al., 1976) and generates a mixture of three pairs of pseudoenantiomers $\left(6^{\mathrm{A}}\right.$-monodeoxy- $6^{\mathrm{A}}$-monoazido- $6^{\mathrm{X}}$ monotosyl- $\beta-C D$, compound $\mathbf{5}$ ). The mixture of primary-side hetero-disubstituted $\beta C D$ was isolated by preparative reversedphase chromatography. The strategy developed for the introduction of the NOPD chromophore in model compound $\boldsymbol{\beta C D}$-NOPD was applied at this stage almost without modifications. The azido moiety remained unaltered both in harsh alkaline conditions (preparation of compound 6) and during the prolonged heating required for the nitrobenzene installation (preparation of compound 7). The most challenging part of the synthetic work was the introduction of the anthracene fluorophore on the NOPDappended $\mathrm{CD}$. A three-step one-pot reaction was carefully designed to fulfill all the necessary requirements. First, a mild reduction method was chosen for the selective reduction of azido group in the presence of the aromatic nitro group. This approach was based on the use of 1,3-propanedithiol and trimethylamine as reduction mixture to obtain the amino-intermediate $\mathbf{7 a}$. Addition of anthracene-9-carboxaldehyde and heating of the reaction mixture resulted in almost complete conversion (90\% conversion rate based on TLC estimation) to anthracene-appended imineintermediate $\mathbf{7 b}$. Portion-wise addition of $\mathrm{NaBH}_{4}$ and prolonged stirring at room temperature afforded anthracene-appended amine-intermediate, compound $\mathbf{8}$, in quantitative conversion. Preparative reversed-phase chromatographic purification allowed the removal of the unreacted compound $7 \mathbf{a}$ and the isolation of targeted compound $\mathbf{8}$. As the CD scaffold is modified simultaneously with two aromatic units, its solubility in aqueous environment is limited. This drawback was overcome by introducing quaternary ammonium bearing moieties in the system, obtaining the desired compound AN-BCD-NOPD.

\subsection{Spectroscopic properties and photochemical behavior}

All compounds synthesized are well soluble in aqueous medium due to the presence of the cationic termination mainly located at the secondary rim of the $\beta C D$ scaffold. The absorption spectrum of AN- $\boldsymbol{\beta C D}$-NOPD ( $\boldsymbol{a}$ in Fig. 1A) exhibits the distinctive spectral features of the anthracene chromophore below $400 \mathrm{~nm}$ and an intense absorption at longer wavelengths extending beyond $450 \mathrm{~nm}$, due to the contribution of the nitroaniline moiety. This 


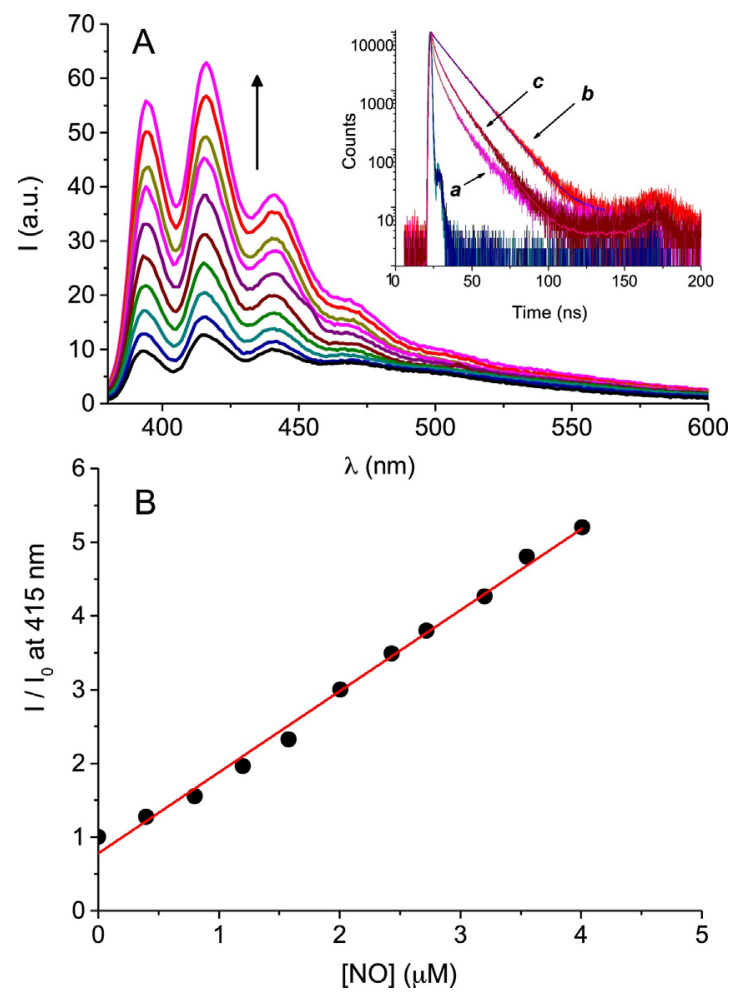

Fig. 4. (A) Fluorescence $\left(\lambda_{\text {exc }}=370 \mathrm{~nm}\right)$ spectral changes observed upon irradiation of an aqueous solution of AN- $\boldsymbol{\beta C D}$-NOPD $(40 \mu \mathrm{M})$ at regular irradiation times of $5 \mathrm{~min}$ (from bottom to top) with visible light $\left(\lambda_{\mathrm{exc}}=405 \mathrm{~nm}\right.$ ). The inset shows the fluorescence decay and the related multiexponential fitting for solutions of $\mathbf{A N}$ $\boldsymbol{\beta C D}$-NOPD $(\boldsymbol{a})$, the model compound AN- $\boldsymbol{\beta C D}(\boldsymbol{b})$ and AN- $\boldsymbol{\beta C D}$-NOPD after $45 \mathrm{~min}$ irradiation with visible light $(\boldsymbol{c}) ; \lambda_{\mathrm{exc}}=370 \mathrm{~nm} ; \lambda_{\mathrm{em}}=420 \mathrm{~nm}$. (B) Correlation of the fluorescence increase observed in Fig. $4 \mathrm{~A}$ and the concentration of NO released from the same solution ( $I$ and $I_{0}$ represent the fluorescence intensities at $420 \mathrm{~nm}$ after each irradiation time and that of the non-irradiated solution, respectively).

spectrum reflects quite well the sum absorption spectrum of the independent model compound AN-BCD and $\boldsymbol{\beta C D}$-NOPD $(\boldsymbol{b}$ and $\boldsymbol{c}$ in Fig. 2A), ruling out any significant interaction between the anthracene and nitroaniline chromophores in the ground state. On the other hand, the intense fluorescence emission of the anthracene fluorophore observed in the case of AN-BCD ( $\boldsymbol{d}$ in Fig. 2) is significantly quenched in the bichromophoric conjugate

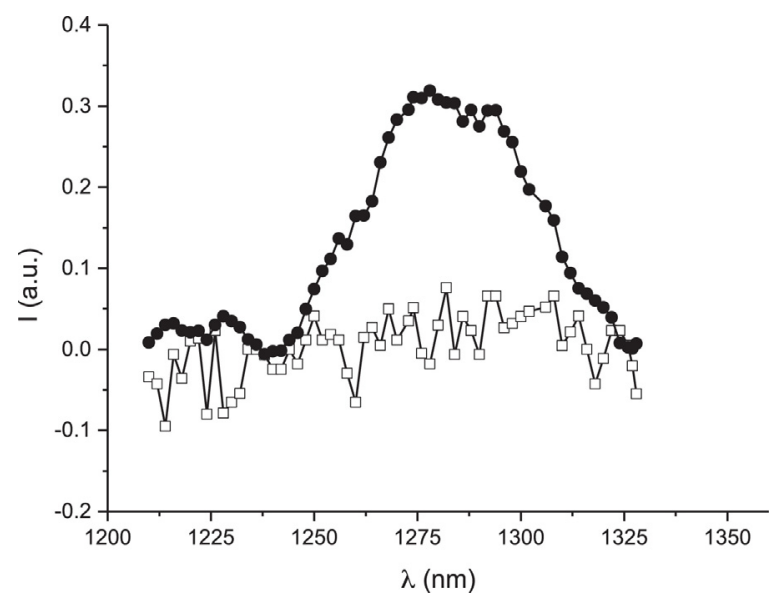

Fig. 5. Luminescence spectra detected upon $370 \mathrm{~nm}$ light excitation of a $\mathrm{D}_{2} \mathrm{O}$

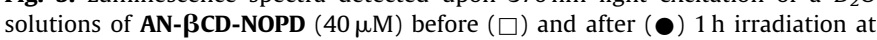
$\lambda_{\text {exc }}=405 \mathrm{~nm}$.

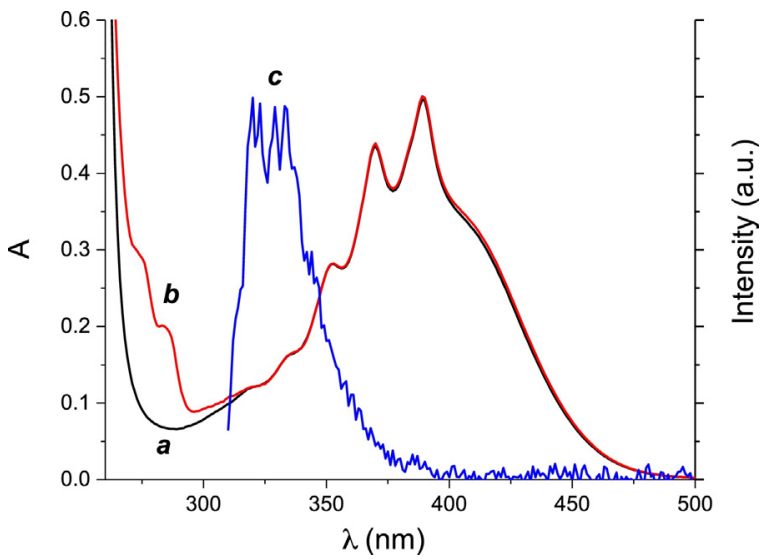

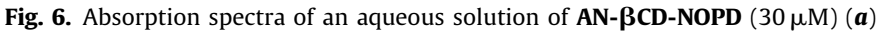
and its complex with naphthalene (b) (left $Y$-axis). Fluorescence emission spectrum (c) observed upon excitation at $\lambda_{\text {exc }}=280 \mathrm{~nm}$ of the sample $\boldsymbol{b}$ (right $Y$-axis).

AN-BCD-NOPD ( $\boldsymbol{e}$ in Fig. 2B), suggesting a remarkable interaction between the two chromophores in the excited state. According to what already demonstrated in our previous studies (Vittorino et al., 2011; Kirejev et al., 2014), this drastic fluorescence quenching can be explained on the basis of a photoinduced energy transfer between the anthracene fluorophore and the nitroaniline components in the conjugate, which is encouraged by the close proximity of the donor and acceptor, both linked at the same rim.

The capability of AN-BCD-NOPD to deliver NO under light stimuli was investigated by the direct and real-time monitoring of this transient species using an ultrasensitive NO electrode which directly reveals NO with nM concentration sensitivity by an amperometric technique (Coneski and Schoenfisch, 2012). The absorption spectral features of AN- $\mathbf{B C D}$-NOPD allow the selective excitation of the NO photoreleaser component of the conjugate at $\lambda_{\text {exc }}>400 \mathrm{~nm}$. The results illustrated in Fig. 3 provide unambiguous evidence that the bichromophoric CD conjugate is very stable in the dark but supplies NO upon illumination with $\lambda_{\text {exc }}=405 \mathrm{~nm}$. Note that, the rate of photorelease is very similar to that observed for an optically matched solution of the model compound $\boldsymbol{\beta C D}$ NOPD.

NO photorelease from AN- $\boldsymbol{\beta C D}$-NOPD is accompanied by dramatic revival of the characteristic emission arising from the anthracene fluorophore. Fig. 4A shows the fluorescence emission spectra observed at regular irradiation times with visible light up to $45 \mathrm{~min}$. This fluorescence restoring is due to the formation of a reaction product formed after the loss of NO that, in contrast to the nitroaniline derivative, is not a good energy acceptor and thus is not able to quench the fluorescence of the anthracene fluorophore. This behavior also reflects in the dynamic of the emission. As shown in the inset in Fig. 4A, the fluorescence decay of AN- $\boldsymbol{\beta C D}$ NOPD (trace $\boldsymbol{a}$ ) was shorter than that of the model compound AN$\boldsymbol{\beta C D}$ (trace $\boldsymbol{b}$ ). However, after $45 \mathrm{~min}$ irradiation of the AN- $\boldsymbol{\beta C D}$ NOPD solution, the fluorescence dynamic become slower (trace $\boldsymbol{c}$ ), according to the revival of the fluorescence. In such a way, the anthracene unit represents an excellent optical reporter for the NO release. In fact, as illustrated in Fig. 4B, we found an excellent linear correlation between the concentration of $\mathrm{NO}$ photogenerated and the increase of the fluorescence intensity of the optical reporter.

Note that, the suppression of the quenching of the excited state of anthracene after NO release reflects also in the capability of this component to photosensitize the formation of singlet oxygen $\left({ }^{1} \mathrm{O}_{2}\right)$, the key species in photodynamic therapy of cancer and bacterial diseases (Hasan et al., 2000). This was directly demonstrated by direct detection through monitoring of the 
typical phosphorescence of ${ }^{1} \mathrm{O}_{2}$ in the near-IR spectral window (Wilkinson et al., 1993). Fig. 5 shows that excitation of AN-BCDNOPD at $\lambda_{\text {exc }}=370 \mathrm{~nm}$ does not lead to any detectable formation of ${ }^{1} \mathrm{O}_{2}$, according to effective quenching occurring of the anthracene by the nitroaniline component. On the other hand, excitation at the same wavelength after irradiating the AN-BCD-NOPD solution for $1 \mathrm{~h}$ at $405 \mathrm{~nm}$, leads to the appearance of the characteristic luminescence signals of ${ }^{1} \mathrm{O}_{2}$ with maximum at ca. 1270 (Fig. 5).

Despite the covalent linking with the two chromophores, the CD cavity of AN- $\boldsymbol{\beta C D}$-NOPD is still able to accommodate hydrophobic guests. This was proven by using naphthalene as a model compound, which is insoluble in water medium. To this end, a methanol solution of naphthalene was prepared. After drying the solvent, the resulting dried sample was added of an aqueous solution of AN- $\boldsymbol{\beta C D}$-NOPD and the sample was stirred for $1 \mathrm{~h}$ at room temperature. The mixture was then filtered and analyzed by UV-Vis absorption. Fig. 6 shows the spectroscopic properties of AN-BCD-NOPD before the addition to the film sample $(\boldsymbol{a})$ and after the filtration procedure $(\boldsymbol{b})$. The presence of the typical structured absorption bands of the naphthalene at ca. 275 and $285 \mathrm{~nm}(\boldsymbol{b})$ provides unambiguous evidence for the encapsulation of the guests within the $\mathrm{CD}$ cavity. Besides, the characteristic fluorescence emission of naphthalene ( $\boldsymbol{c}$ in Fig. 6) further confirms the effective formation of the host-guest supramolecular complex.

\section{Conclusions}

We have devised and synthesized a novel multifunctional CD conjugate, bearing a nitroaniline and an anthracene chromophoric unit, able to incorporate a number of functionalities within the same molecular skeleton. AN-BCD-NOPD is well soluble in water due to the cationic appendages present mainly at the lower rim of the $\mathrm{CD}$ scaffold and is able to deliver NO under the exclusive control of visible light stimuli. NO photorelease restores the fluorescence of the anthracene fluorophore, which acts as an optical reporter of NO concentration. To the best of our knowledge, this represents the first example of NO photodispenser with fluorescence reporting integrated in a CD scaffold. Furthermore, we have demonstrated that the anthracene component is also capable to photogenerate ${ }^{1} \mathrm{O}_{2}$ sequentially to the NO release. The $\mathbf{A N}-\boldsymbol{\beta C D}$-NOPD conjugate is also able to accommodate hydrophobic guests within the $\beta C D$ cavity, as proven by using naphthalene as a model compound. In view of the key role $\mathrm{NO}$ and ${ }^{1} \mathrm{O}_{2}$ plays in photodynamic therapy, this $\beta C D$ derivative represents an intriguing candidate for biopharmaceutical research. Studies in this direction are currently underway in our laboratories.

\section{Acknowledgements}

We thank the Marie Curie Program (FP7-PEOPLE-ITN-2013, CYCLON-HIT 608407) for financial support to MC fellows GB, MPL and DA, and funding of the research. SB thanks for the financial support from NKFIH 109373 and from ÚNKP-16-4 New National Excellence Program of the Ministry of Human Capacities.

\section{Appendix A. Supplementary data}

Supplementary data associated with this article can be found, in the online version, at http://dx.doi.org/10.1016/j. ijpharm.2017.05.023.

\section{References}

Bortolus, P., Monti, S., 1996. Photochemistry in cyclodextrin cavities. Adv. Photochem. 21, 1-133.
Caruso, E.B., Petralia, S., Conoci, S., Giuffrida, S., Sortino, S., 2007. Photodelivery of nitric oxide from water-soluble platinum nanoparticles. J. Am. Chem. Soc. 129, $480-481$.

Coneski, P.N., Schoenfisch, M.H., 2012. Nitric oxide release: part III. Measurement and reporting. Chem. Soc. Rev. 41, 3753-3758.

Davis, M.E., Brewster, M.E., 2004. Cyclodextrin-based pharmaceutics: past, present and future. Nat. Rev. Drug Discov. 3, 1023-1035.

Ford, P.C., 2008. Polychromophoric metal complexes for generating the bioregulatory agent nitric oxide by single- and two-photon excitation. Acc. Chem. Res. 41, 190-200.

Ford, P.C., 2013. Photochemical delivery of nitric oxide. Nitric Oxide 34, 56-64.

Fraix, A., Marino, N., Sortino, S., 2016. Phototherapeutic release of nitric oxide with engineered nanoconstructs. Top. Curr. Chem. 370, 225-257.

Fry, N.L., Mascharak, P.K., 2011. Photoactive ruthenium nitrosyls as NO donors: how to sensitize them toward visible light. Acc. Chem. Res. 44, 289-298.

Hasan, T., Moor, A.C.E., Ortel, B., 2000. Cancer Medicine, 5th ed. Decker BC Inc., Hamilton, Ontario, Canada.

Ignarro, L.J., 2009. Special journal issue on nitric oxide chemistry and biology. In: Ignarro, L.J. (Ed.), Arch. Pharmacal Res., .

Ignarro, L.J., 2010. Nitric Oxide: Biology and Pathobiology. Elsevier Inc..

Jia, Q., Janczuk, A.J., Cai, T., Xian, M., Wen, Z., Wang, P.G., 2002. NO donors with anticancer activity. Expert Opin. Ther. Patents 12, 819-826.

Jicsinszky, L., Iványi, R., 2001. Catalytic transfer hydrogenation of sugar derivatives. Carbohydr. Polym. 45, 139-145.

Kirejev, V., Kandoth, N., Gref, R., Ericson, M.B., Sortino, S., 2014. A polymer-based nanodevice for the photoregulated release of NO with two-photon fluorescence reporting in skin carcinoma cells. J. Mater. Chem. B 2, 1190-1195.

Loftsson, T., Douchene, D., 2007. Cyclodextrins and their pharmaceutical applications. Int. J. Pharm. 329, 1-11.

Matsui, Y., Yokoi, T., Mochida, K., 1976. Catalytic properties of Cu(II) complex with a modified cyclodextrin. Chem. Lett. 5, 1037-1040.

Mazzaglia, A., Micali, Monsù, L., Scolaro, L., 2006. Cyclodextrin Materials Photochemistry. Photophysics and Photobiology. Elsevier, pp. 203-222 and ref therein.

Mazzaglia, A., Sciortino, M.T., Kandoth, N., Sortino, S., 2012. Cyclodextrin-based nanoconstructs for photoactivated therapies. J. Drug Deliv. Sci. Technol. 22, 235 242.

Monti, S., Sortino, S., 2002. Photoprocesses of photosensitizing drugs within cyclodextrin cavities. Chem. Soc. Rev. 31, 287-300.

Mourtzis, N., Eliadou, K., Aggelidou, C., Sophianopoulou, V., Mavridis, I.M., Yannakopoulou, K., 2007. Per(6-guanidino-6-deoxy)cyclodextrins: synthesis, characterisation and binding behaviour toward selected small molecules and DNA. Org. Biomol. Chem. 5, 125-131.

Mourtzis, N., Paravatou, M., Mavridis, I.M., Roberts, M.L., Yannakopoulou, K., 2008 Synthesis, characterization, and remarkable biological properties of cyclodextrins bearing guanidinoalkylamino and aminoalkylamino groups on their primary side. Chem. Eur. J. 14, 4188-4200.

Pellois, J.-P., Hahn, M.E., Muir, T.W., 2004. Simultaneous triggering of protein activity and fluorescence. J. Am. Chem. Soc. 126, 7170-7171.

Piras, L., Theodossiou, T.A., Manouilidou, M.D., Lazarou, Y.G., Sortino, S., Yannakopoulou, K., 2013. S-Nitroso-b-cyclodextrins as new bimodal carriers: preparation, detailed characterization, nitric-oxide release, and molecular encapsulation. Chem. Asian J. 8, 2768-2778.

Riccio, D.A., Schoenfisch, M.H., 2012. Nitric oxide release: part I. Macromolecular scaffolds. Chem. Soc. Rev. 41, 3731-3741.

Seabra, A.B., Durán, N., 2010. Nitric oxide-releasing vehicles for biomedical applications. J. Mater. Chem. 20, 1624-1637.

Sortino, S., 2010. Light-controlled nitric oxide delivering molecular assemblies. Chem. Soc. Rev. 39, 2903-2913.

Ueno, A., Breslow, R., 1982. Selective sulfonation of a secondary hydroxyl group of $\beta$-cyclodextrin. Tetrahedron Lett. 23, 3451-3454.

Veldhuyzen, W.F., Nguyen, Q., McMaster, G., Lawrence, D.S., 2003. A light-activated probe of intracellular protein kinase activity. J. Am. Chem. Soc. 125, 1335813359.

Vittorino, E., Sciortino, M.T., Siracusano, G., Sortino, S., 2011. Light-activated release of nitric oxide with fluorescence reporting in living cells. ChemMedChem 6, 1551-1554.

Wang, P.G., Xian, M., Tang, X., Wu, X., Wen, Z., Cai, T., Janczuk, A.J., 2002. Nitric oxide donors: chemical activities and biological applications. Chem. Rev. 102, 10911134.

Wang, P.G., Cai, T.B., Taniguchi, N., 2005. Nitric Oxide Donors for Pharmaceutical and Biological Applications. Wiley-VCH Verlag GmbH \& Co. KGaA, Weinheim, Germany.

Weinstain, R., Segal, E., Fainaro, R.S., Shabat, D., 2010. Real-time monitoring of drug release. Chem. Commun. 46, 553-555.

Wilkinson, F., Helman, W.P., Ross, A.B., 1993. Quantum yields for the photosensitized formation of the lowest electronically excited singlet state of molecular oxygen in solution. J. Phys. Chem. Ref. Data 22, 113-262.

Yannakopoulou, K., Jicsinszky, L., Aggelidou, C., Mourtzis, N., Robinson, T.M., Yohannes, A., Nestorovich, E.M., Bezrukov, S., Karginov, V.A., 2011. Symmetry requirements for effective blocking of pore forming toxins: comparative study with $\alpha$-, $\beta$-, and $\gamma$-cyclodextrin derivatives. Antimicrob. Agents Chemother. 55, 3594-3597. 